\title{
DESEMPENHO DE NOVILHOS MESTIÇOS NELORE SUPLEMENTADOS EM PASTAGEM COM DIFERENTES NÍVEIS DE CONCENTRADO NO PERÍODO SECO DO ANO ${ }^{1}$
}

\author{
Levels of concentrate for grazing nelore crossbred steers in the dry period of the year
}

\author{
Afranio Afonso Ferrari Baião ${ }^{2}$, Ivo Francisco de Andrade³, Edinéia Alves Moreira Baião ${ }^{4}$ Leonardo Alves Baião ${ }^{6}$, \\ Juan Ramón Olalquiaga Pérez ${ }^{3}$, Carlos Alberto Pereira de Rezende ${ }^{3}$, Joel Augusto Muniz ${ }^{5}$, \\ Carlos Alexandre Jeronimo Vieira ${ }^{7}$, George Delmond Bueno ${ }^{7}$
}

\begin{abstract}
RESUMO
No Brasil, a engorda de animais suplementados em pastagem na seca, nos últimos anos, representa a maior parte dos animais terminados nesta época do ano. Com este trabalho objetivou-se avaliar o efeito de níveis crescentes de concentrado na engorda de novilhos mestiços nelore, suplementados em pastagem no período seco do ano. O experimento foi realizado no Setor de Bovinocultura do Departamento de Zootecnia da Universidade Federal de Lavras - UFLA, entre os meses de junho a setembro de 2001. Foram utilizados 24 bovinos mestiços nelore, machos, inteiros, com idade média de 30 meses e peso vivo médio de $295 \mathrm{Kg}$. Durante o período experimental, os animais foram manejados em pastagem de Brachiaria brizanta cv. Marandu com área de 11,5 ha e disponibilidade média de 7,635 e 3,495 kgMS.ha-1 ${ }^{-1}$ no início e no final do período, respectivamente. Os tratamentos constituíram-se de níveis crescentes de concentrado, calculados como percentual do peso vivo, como segue: $\mathrm{T}_{1}-0,0 \% ; \mathrm{T}_{2}-0,4 \% ; \mathrm{T}_{3}-0,8 \% ; \mathrm{T}_{4} \mathbf{-}^{-} 1,2 \%$. $\mathrm{O}$ experimento foi delineado em blocos casualizados, com o peso vivo inicial como fator de blocagem, sendo 6 blocos e 4 tratamentos, totalizando 24 parcelas experimentais. Para análise dos dados utilizou-se o software estatístico Sistema de Análise de Variância de Dados Balanceados (SISVAR). Os resultados foram os seguintes: houve efeito significativo, com ajuste linear, dos níveis de concentrado no ganho de peso diário $(\mathrm{P}<0,05)$, com ponto de máximo de $0,87 \%$ do PV. Com base na análise dos dados, conclui-se que a terminação de novilhos nelores, suplementados a pasto, é técnica e economicamente viável, com melhores resultados ao nível de $0,76 \%$ do PV.
\end{abstract}

Termos para indexação: Brachiária brizanta cv. Marandu, ganho de peso, mestiços nelore, nível de suplementação.

\begin{abstract}
In Brazil, the fattening of suplemented grazing animals in the last years accounted for most of the finishing animals in this time of the year. This work had the objective to evaluate the effect of increasing levels of concentrate in the fattening of crossbreed bullocks of Nelore breed grazing suplemented Brachiaria brizanta cv. Marandu pasture in the dry period of the year. The experiment was conducted in the Beef Cattle Division of the Animal Science Department at Universidade Federal de Lavras, Minas Gerais, from June to September of 2001. It was used 24 crossbreed Nelore cattle, males, not castrated, averaging 30 months old and with the means of live weight of $295 \mathrm{Kg}$. During the experimental period, the animals were housed in a sealed pasture of Brachiária brizanta cv. Marandu, with an area of 11,50 ha and the average availability of 7,635 and 3,495 kgDM/ha at the begining and at the end of the period, respectively. The treatments were constituted of increasing levels of concentrate calculated as percentage of the body live weight, as follow: T1 - 0\%; T2 - 0,4\%; T3 - 0,8\%; T4-1,2\%. The experimental design was a randomized complete block design with the initial live weight as the blocking factor: 6 blocks, 4 treatments, totaling 24 experimental units. For the data analysis the statistical software SISVAR was used (Variance Analysis System of Balanced Data). There was significant effect (with linear adjustment) of the concentrate levels in the daily weight gain $(\mathrm{P}<0,05)$, with point of maximum of $0,87 \%$ of BLW. Based on the data analysis, it was concluded that the finishing of Nelore bullocks, grazing suplementated Brachiaria brizanta cv. Marandu pasture, is technically and economically viable with better results at the level of $0,76 \%$ of BLW.
\end{abstract}

Index Terms: brachiaria brizanta cv. Marandu, live weight gain, crossbreed of nelore breed, level of the suplementation.

(Recebido para publicação em 10 de maio de 2002 e aprovado em 27 de setembro de 2004)

\section{INTRODUÇÃO}

O Brasil possui o maior rebanho bovino comercial do mundo, com um efetivo de 163,470 milhões de cabeças, e ocupa a segunda posição na produção de carne, perdendo apenas para os Estados Unidos, com 6,182 milhões de toneladas (ANUALPEC, 2001).

Embora desfrute de altos números absolutos, a

\footnotetext{
1 Extraído da Dissertação de Mestrado apresentada pelo primeiro autor à Universidade Federal de Lavras.

2 Engenheiro Agrônomo, aluno do Curso de Pós-graduação em Zootecnia da Universidade Federal de Lavras - Cx. P. 3037 - $37.200-000$ - Lavras, MG a2fbaiao@yahoo.com.br

${ }^{3}$ Professor da Universidade Federal de Lavras - Departamento de Zootecnia - Cx. P. 3037 - 37.200-000 - Lavras, MG.

${ }^{4}$ Engenheira Agrônoma, aluna Curso de Pós-graduação em Zootecnia da Universidade Federal de Lavras - Cx. P. 3037 - $37.200-000$ - Lavras, MG.

${ }^{5}$ Professor da Universidade Federal de Lavras - Departamento de Ciências Exatas - Cx. P. 3037 - $37.200-000$ - Lavras, MG.

${ }^{6}$ Aluno do Curso de graduação em Ciências Biológicas da Universidade Federal do Espírito Santo.

${ }^{7}$ Aluno do Curso de graduação em Zootecnia da Universidade Federal de Lavras.
} 
pecuária bovina de corte brasileira apresenta baixo desempenho, demonstrado por seus índices zootécnicos: baixo percentual de natalidade, alto percentual de mortalidade até a desmama, idade elevada ao primeiro parto das novilhas e de abate dos machos.

O cenário atual do mercado de carnes pressupõe a evolução dos sistemas de produção no sentido de buscar eficiência e qualidade do produto, visando obter competitividade e sustentabilidade. Assim, a pecuária brasileira deve estabelecer as bases de um desenvolvimento sustentável, que se caracterize por ser economicamente menos vulnerável, socialmente mais justo e ecologicamente correto.

As pastagens constituem a principal fonte de alimentação dos bovinos no Brasil e, na maioria dos casos, constituem a sua única fonte de alimento. Elas representam também a forma mais viável e prática de alimentação dos rebanhos.

Em função da existência de duas estações climáticas bem definidas, uma chuvosa, que vai normalmente de outubro/novembro a abril/maio, e outra seca, que vai de abril/ maio a outubro/ novembro, a nutrição animal em condições de pastagens oscila muito.

No período chuvoso, quando a disponibilidade e a qualidade da forragem, se não boas, são pelo menos razoáveis, os animais apresentam ganho de peso. Já na seca, tanto a qualidade quanto a disponibilidade de forragem são baixas e os animais mal conseguem manter o seu peso, não sendo rara a perda de peso. Devido a esse desequilíbrio na nutrição animal, a produção em regime de pastagens é insatisfatória.

O desenvolvimento de estratégias para a produção bovina no período seco do ano é fundamental para garantir maior produtividade da pecuária de corte brasileira. A suplementação a pasto para engorda de bovinos no período seco do ano surge, portanto, como uma alternativa ao confinamento tradicional, vem crescendo muito nos últimos anos e necessita de mais pesquisas por ser um grande desafio à nutrição de bovinos de corte.

Conduziu-se este trabalho com objetivo de determinar o nível de suplementação concentrada para recria de novilhos nelores, mantidos em pastagens de Brachiaria brizanta cv. Marandu, no período seco do ano.

\section{MATERIAL E MÉTODOS}

\section{Local e Condições Climáticas}

O experimento foi realizado no Setor de Bovinocultura do Departamento de Zootecnia, da Universidade Federal de Lavras que se localiza na região sul do Estado de Minas Gerais, a 918 metros de altitude, tendo como coordenadas geográficas $21^{\circ} 14^{\prime}$ de latitude Sul e $45^{\circ} 00^{\prime}$ de longitude Oeste de Greenwich (CASTRO NETO et al., 1980). O clima é do tipo CWb, segundo a classificação Köeppen (1948), possuindo duas estações distintas: uma chuvosa, que vai de novembro a abril e outra seca, que se prolonga de maio a outubro (OMETTO, 1981). A precipitação média anual é de $1.492 \mathrm{~mm}$ e as temperaturas médias das máximas e mínimas anual são respectivamente, $26^{\circ} \mathrm{C}$ e $14^{\circ} \mathrm{C}$ (VILELA \& RAMALHO, 1979).

Os dados de precipitação pluviométrica, temperatura e umidade relativa do ar durante os períodos pré-experimental e experimental são apresentados na Tabela 1.

TABELA 1 - Temperaturas médias mensais de máximas e mínimas, precipitação e umidade relativa do ar (URA) no período de abril a setembro de 2001.

\begin{tabular}{lcccc}
\hline Período & $\begin{array}{c}\text { Temp. } \\
\text { mínima }\left({ }^{\circ} \mathbf{C}\right)\end{array}$ & $\begin{array}{c}\text { Temp. } \\
\text { máxima }\left({ }^{\circ} \mathbf{C}\right)\end{array}$ & $\begin{array}{c}\text { Prec. } \\
\text { Pluviométrica }(\mathbf{m m})\end{array}$ & $\begin{array}{c}\text { URA } \\
(\boldsymbol{\%})\end{array}$ \\
\hline Abril & 16,45 & 29,41 & 17,60 & 68,90 \\
Maio & 13,16 & 25,37 & 48,30 & 72,30 \\
Junho & 11,88 & 25,54 & 00,00 & 69,20 \\
Julho & 11,91 & 26,15 & 00,00 & 64,12 \\
Agosto & 12,38 & 26,21 & 63,20 & 59,99 \\
Setembro & 14,05 & 29,90 & 46,10 & 67,73 \\
\hline
\end{tabular}

Fonte: Estação Agrometeorológica-DEG/UFLA (2001). 


\section{Período Experimental, Animais e Instalações}

A duração do trabalho foi de 114 dias, compreendidos entre 27 de maio e 21 de setembro de 2001. Neste período, os primeiros 30 dias foram destinados à adaptação dos animais, e o período experimental teve a duração de 84 dias a partir de 28 de junho de 2001.

Foram utilizados 24 novilhos mestiços nelores, inteiros, com idade média de 30 meses e peso vivo médio inicial de $295 \mathrm{Kg}$, vacinados previamente contra aftosa e raiva, vermifugados, identificados por brincos numerados na orelha esquerda, provenientes de rebanhos comerciais da região.

Os animais foram mantidos em uma pastagem de Brachiaria brizanta cv. Marandu vedada em dezembro de 2000, com área total de 11,5 ha. Na área experimental, como não havia aguada natural, foi instalado um bebedouro artificial, bem como cocho coletivo para mistura mineral, com capacidade para que todos os animais pudessem suprir suas necessidades.

Para a condução deste trabalho, foi utilizada uma instalação anexa à área de pastagem (aproximadamente 20 $\mathrm{m}$ de distancia), constituindo-se de um curral de contenção e 18 baias com cochos individuais, medindo $2 \mathrm{~m}$ de comprimento x $1 \mathrm{~m}$ de largura $\left(2 \mathrm{~m}^{2}\right)$ cada uma, e uma balança tipo brete com capacidade para $1.500 \mathrm{~kg}$

Os 24 animais eram conduzidos diariamente até o curral de contenção, onde somente 18 animais eram contidos em baias individuais e recebiam seus tratamentos enquanto os 6 restantes aguardavam no curral de contenção. Quando os animais que estavam recebendo a suplementação finalizavam o consumo, os mesmos eram reconduzidos, juntamente com os animais que não recebiam suplemento, para a pastagem onde permaneciam até a próxima suplementação no dia seguinte.
As sobras existentes eram retiradas e pesadas individualmente para posterior cálculo do consumo de cada animal.

\section{Tratamentos}

Os tratamentos consistiram do fornecimento diário de níveis crescentes de concentrado protéico-energético, nos níveis de $0 ; 0,4 ; 0,8$ e 1,2\% do peso vivo dos animais em concentrado. A comparação entre os tratamentos foi feita levando-se em conta o desempenho dos animais em um delineamento de blocos inteiramente casualizados (DBC) com 6 repetições.

A ração experimental, fornecida aos animais, era constituída de sorgo desintegrado com panícula, casca de café, farelo de soja, farelo de algodão e farelo de arroz. A ração foi formulada segundo as normas do NRC (1996) para um ganho de peso vivo diário de $1 \mathrm{~kg}$.

A composição do suplemento e os teores de matéria seca (MS), proteína bruta $(\mathrm{PB})$, nutrientes digestíveis totais (NDT), cálcio $(\mathrm{Ca})$, fósforo $(\mathrm{P})$ e fibra em detergente neutro (FDN) dos ingredientes e do suplemento, na Tabela 2.

\section{Coleta de dados e análises químicas}

\section{Animais}

Os animais eram conduzidos ao curral de manejo todos os dias às 12:00 horas para serem suplementados, $\mathrm{e}$ a cada 7 dias, antes de receberem a ração experimental, eram pesados individualmente.

As pesagens eram feitas sempre às 12:00 horas, $\mathrm{e}$ logo após, os animais eram conduzidos às baias individuais para receberem os suplementos e, posteriormente, reconduzidos à pastagem, totalizando 12 pesagens durante todo o período experimental.

TABELA 2 - Composição Percentual do Suplemento, Teores Médios de Matéria Seca (MS), Proteína Bruta (PB), Nutrientes Digestíveis Totais (NDT), Cálcio (Ca), Fósforo (P) e Fibra em Detergente Neutro (FDN) em cada um dos ingredientes da dieta animal (\%) e no suplemento (\%).

\begin{tabular}{lccccccc}
\hline Ingredientes & Suplemento* $^{*}$ & MS & PB & NDT $* *^{*}$ & Ca & P & FDN \\
\hline Casca de Café & 44,28 & 88,00 & 11,00 & 54,00 & 0,26 & 0,09 & 70,00 \\
Farelo de Soja & 9,67 & 90,00 & 49,00 & 84,00 & 0,29 & 0,70 & 15,00 \\
Farelo de Algodão & 2,48 & 91,00 & 45,00 & 80,00 & 0,22 & 1,21 & 25,00 \\
Farelo de Trigo & 15,39 & 89,00 & 15,00 & 67,00 & 5,00 & 0,80 & 12,00 \\
Sorgo (Panícula) & 28,18 & 92,00 & 7,00 & 84,00 & 0,29 & 0,14 & 60,00 \\
\hline Suplemento & 100,00 & 90,76 & 15,00 & 68,00 & 1,00 & 0,30 & 51,83 \\
\hline
\end{tabular}

Análises realizadas no Laboratório de Nutrição Animal do departamento de Zootecnia/ UFLA.

* \% na Matéria Seca.

** Valores estimados pelo NRC (1996).

Ciênc. agrotec., Lavras, v. 29, n. 6, p. 1258-1264, nov./dez., 2005 


\section{Forragem}

A área de pastagem utilizada foi vedada em dezembro de 2000, sendo utilizada inicialmente no mês de maio de 2001 para a fase pré-experimental.

Foram realizadas amostragens da forragem a cada 14 dias, totalizando 6 amostras durante todo o período experimental. Essas amostragens foram efetuadas com o objetivo de obter a composição bromatológica e a variação da forragem em função do tempo, bem como a disponibilidade de matéria seca (MS) no decorrer do experimento. Essas amostragens foram feitas em 30 pontos da pastagem, rente ao solo, utilizando um quadrilátero de $1,00 \times 1,00 \mathrm{~m}\left(1,00 \mathrm{~m}^{2}\right)$, lançado aleatoriamente dentro da pastagem, as amostras eram pesadas e delas era retirada uma amostra composta.

As amostras foram levadas ao Laboratório de Nutrição Animal do Departamento de Zootecnia para análises bromatológicas. Inicialmente, foram secas ao ar em estufa de ventilação forçada a $65{ }^{\circ} \mathrm{C}$, por 72 horas ou terem peso constante, para determinação da matéria préseca (ASA). Em seguida, as amostras foram moídas em moinho de faca tipo Willey de 30 "mesh", para posterior determinação da matéria seca a $105^{\circ} \mathrm{C}$ (ASE), proteína bruta, cinzas, cálcio , fósforo (SILVA, 1998), fibra em detergente neutro e fibra em detergente ácido (SOEST et al., 1991). Com a determinação da matéria seca das amostras, fez-se o cálculo da disponibilidade de matéria seca (MS).

\section{Relação Receita:Despesa}

Para relação receita: despesa, os cálculos foram levantados de modo a refletir, de maneira mais próxima, o que seria feito caso o produtor implantasse, na propriedade, neste ano, nestas mesmas condições, um sistema semelhante.

Os preços dos alimentos concentrados (farelo de trigo, farelo de algodão, farelo de soja), vermífugos, medicamentos e sal mineral foram levantados na Cooperativa Agrícola Alto Rio Grande (CAARG), do município de Lavras, considerando que o produtor faria opção por comprar os alimentos estrategicamente no mês do ano em que estes estivessem mais baratos. $\mathrm{O}$ valor do sorgo desintegrado com panícula foi calculado usando o preço do sorgo no mês de Maio/2001 (em pesquisa feita entre produtores de sorgo da região), considerando que uma tonelada do produto (sorgo desintegrado com panícula) equivale a 10 sacas de sorgo grão. Para casca de café, foi considerado apenas o preço do frete do transporte da beneficiadora de café até a propriedade. O preço do boi foi estimado em $\mathrm{R} \$ 42,00 /$ arroba, vigente no município de Lavras no mês de setembro/2001.

A mão-de-obra foi estimada na base de meio salário mínimo, considerando que o funcionário precisaria de apenas meio dia para tratar os animais e a outra metade do salário seria paga por outras atividades da fazenda. $\mathrm{O}$ custo da alimentação volumosa foi estimada mediante a base estabelecida pela prática na região de aluguel de pastagem, que é de um quilo de carne de primeira por animal, por mês, sendo usado como referência o mês de junho, logo ao início do experimento.

A receita foi calculada utilizando o preço real de venda destes animais por arroba, multiplicado pelo seu peso na mesma unidade. A divisão da receita total pelo custo total resultou na relação citada.

\section{Delineamento Experimental}

Os tratamentos foram constituídos por quatro níveis de suplementação em porcentagem (\%) do peso vivo $(0,0$; $0,4 ; 0,8$ e 1,2$)$. Os suplementos foram ajustados a cada sete dias, após a realização das pesagens. Utilizou-se o delineamento em blocos casualizados (DBC) com seis repetições, procurando-se controlar o peso dos animais.

A parcela experimental constituiu-se de um animal.

A análise estatística foi realizada utilizando-se o software estatístico "Sistema de Análise de Variância de Dados Balanceados" (SISVAR), de acordo com Ferreira (2000).

Os tratamentos foram comparados por meio da análise de regressão, considerando os níveis de suplementos.

O modelo estatístico do experimento foi:

$$
\mathbf{Y}_{\mathrm{ij}}=\mu+\mathbf{t}_{\mathbf{i}}+\mathbf{b}_{\mathbf{j}}+\mathbf{e}_{\mathrm{ij}}
$$

Em que:

$\mathbf{Y}_{\mathrm{ij}}=$ valor da parcela que recebeu o tratamento i no bloco j;

$\mathbf{m}=$ constante associada a todas observações;

$\mathbf{t}_{\mathrm{i}}=$ efeito do tratamento $\mathrm{i}$, com $\mathrm{i}=1,2,3,4$;

$\mathbf{b}_{\mathbf{j}}=$ efeito do bloco $\mathrm{j}$, com $\mathrm{j}=1,2,3,4,5,6$;

$\mathbf{e}_{\mathrm{ijj}}=$ o erro experimental associado a $\mathbf{Y}_{\mathrm{ij}}$, que por hipótese. tem distribuição normal com média zero e variância de $\mathrm{s}^{2}$.

\section{RESULTADOS E DISCUSSÃO}

\section{Disponibilidade e composição bromatológica da forragem}

A disponibilidade de matéria seca é apresentada na Tabela 3, e a composição bromatológica da forragem é apresentada na Tabela 4. 
BAIÃO, A. A. F. et al.

TABELA 3 - Disponibilidade média de matéria seca (MS) no período de 01 de maio a 21 de setembro de 2001.

\begin{tabular}{lccccc}
\hline & \multicolumn{5}{c}{ Mês/ano } \\
\cline { 2 - 6 } MS (t/ha) & Maio & Junho & Julho & Agosto & Setembro \\
\cline { 2 - 6 } & 10,143 & 7,635 & 5,653 & 4,582 & 3,495 \\
\hline
\end{tabular}

TABELA 4 - Composição bromatológica da forragem.

\begin{tabular}{lcccc}
\hline \multirow{2}{*}{ Parâmetro } & \multicolumn{3}{c}{ Data de amostragem } \\
& $\mathbf{2 8 / 0 6}$ & $\mathbf{2 6 / 0 7}$ & $\mathbf{2 4 / 0 8}$ & $\mathbf{2 1 / 0 9}$ \\
\hline MS (\%) & 80,35 & 85,28 & 93,43 & 90,14 \\
PB $^{*}$ & 5,32 & 4,26 & 2,98 & 3,48 \\
MM $^{*}$ & 5,89 & 4,65 & 4,38 & 4,51 \\
FDN $^{*}$ & 72,23 & 75,52 & 82,67 & 81,21 \\
FDA $^{*}$ & 45,68 & 46,28 & 49,77 & 49,08 \\
a $^{*}$ & 0,51 & 0,49 & 0,43 & 0,45 \\
P* $^{*}$ & 0,28 & 0,28 & 0,25 & 0,26 \\
\hline
\end{tabular}

*\% na MS.

\section{Consumo}

Durante o período pré-experimental e durante a $1^{\mathrm{a}}$ semana do período experimental, os animais consumiram normalmente o suplemento nos níveis previstos, mas a partir da $2^{\mathrm{a}}$ semana um dos animais que recebia o tratamento $2(0,4 \% \mathrm{PV})$, dois animais que recebiam o tratamento 3 $(0,8 \% \mathrm{PV})$ e todos os animais que recebiam o tratamento 4 $(1,2 \% \mathrm{PV})$ passaram a não consumir totalmente o suplemento. Estes fatos ocorreram, provavelmente, pelo aumento da FDN da pastagem no decorrer do experimento (Tabela 4) e pelo fornecimento do suplemento apenas uma vez por dia, quando o ideal seria o fornecimento dividido em duas vezes (quando a quantidade de suplemento a ser fornecida for acima de $2 \mathrm{~kg} / \mathrm{dia}$ ). Após o cálculo do consumo ao final do experimento os novos níveis dos tratamentos ficaram da seguinte forma: $\mathrm{T} 1=0,0 \% ; \mathrm{T} 2=$ $0,37 \%$; $\mathrm{T} 3=0,76 \%$ e $\mathrm{T} 4=0,87 \%$ do peso vivo.

\section{Ganho de peso diário}

Observou-se diferença significativa ( $\mathrm{p} \mathrm{d}$ " 0,05) para ganho de peso diário entre os tratamentos. A análise de variância do ganho de peso diário revelou uma resposta linear (p d" 0,05 ) em relação aos níveis de concentrado (Figura 1).

O estudo dessa regressão linear permite dizer que quanto maior o nível de concentrado, maior o ganho de peso diário (dentro do intervalo estudado de 0 a $0,87 \%$ $\mathrm{PV}$ ), portanto o fornecimento de $0,87 \%$ do PV foi o nível de melhor resposta, o que resultou num ganho de peso de $0,462 \mathrm{~kg}$ por dia.

A melhor resposta em ganhos adicionais observada neste trabalho foi de $0,87 \%$ do PV, este valor é semelhante ao resultado verificado por Euclides et al. (1998), de 0,8\% do PV, bem como por Almeida \& Azevedo (1999), de $1 \%$ do PV, Paulino (1999), entre 0,8 e $1 \%$ do PV, e Bomfim (2000) de 0,84\% do PV. O ganho de peso diário observado para essa quantidade de concentrado $(0,462$ $\mathrm{kg}$ ) é semelhante as observações de Boin \& Tedeschi (1997), de 0,5 kg/dia e inferiores as observadas por Almeida \& Azevedo (1999), de 0,72 kg/dia, e por Bomfim (2000), de $0,6 \mathrm{~kg} / \mathrm{dia}$.

\section{Conversão alimentar em ganho de peso}

Sendo $\mathrm{X}$ a quantidade de alimento fornecida ao animal e Y a correspondente transformação produzida, a medida natural de eficiência relativa, conversão alimentar (CA) e eficiência alimentar (EA) são traduzidas pelos quocientes $\mathrm{CA}=\mathrm{X} / \mathrm{Y}$ ou $\mathrm{EA}=\mathrm{Y} / \mathrm{X} \operatorname{com} \quad \mathrm{X}=\mathrm{x}>0 ; \mathrm{Y}=$ $y>0$. Essa definição tem o inconveniente de não se aplicar aos casos eventuais que possam ocorrer em certos tipos de experimentos em que $\mathrm{x}=0$ ou y d" 0 (GUIDONI, 1994). Como ocorreram os dois fatos neste experimento $(\mathrm{x}=0 \mathrm{e}$ y $<0$ ), não é recomendado fazer análise estatística dos dados de CA e EA, portanto apresenta-se somente as médias das Conversões Alimentares de cada tratamento (Tabela 5). 


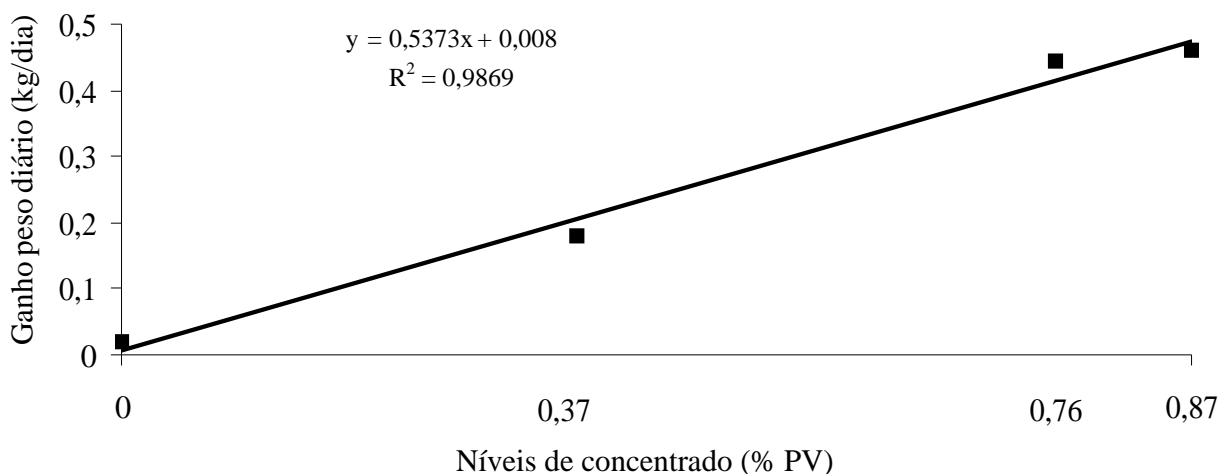

FIGURA 1 - Efeito dos níveis de concentrado no ganho de peso diário.

TABELA 5 - Valores da conversão alimentar total (forragem + concentrado).

\begin{tabular}{cc}
\hline Níveis do Suplemento (\% PV) & Conversão $(\mathbf{k g} / \mathbf{k g})$ \\
\hline 0,00 & 183,79 \\
0,37 & 21,96 \\
0,76 & 9,19 \\
0,87 & 9,09 \\
\hline
\end{tabular}

A conversão depende da resposta de ganhos de peso ao consumo da forragem e concentrado e são fatores importantes na determinação do custo do ganho e da rentabilidade da suplementação (WAGNER et al., 1995).

\section{Relação receita:despesa}

Os valores médios da receita ( $\mathrm{R} \$$ animal), despesa ( $\mathrm{R} \$$ /animal) e a relação receita:despesa são apresentados na Tabela 6.

Houve diferença estatística $(\mathrm{P}>0,05)$ conforme análise de variância entre as relações receita:despesa nos tratamentos, que respondeu de forma linear (Figura 2).

Como o ganho de peso total por animal no tratamento T4 (0,87\% PV) foi de $38,833 \mathrm{~kg}$, a um custo de $\mathrm{R} \$ 50,76$, e uma receita de $\mathrm{R} \$ 54,37$, isto representa uma renda líquida de $\mathrm{R} \$ 3,61$ (três reais e sessenta e um centavos), a qual comparada com ao prejuízo do tratamento com $\mathrm{T} 1$ ( $0 \% \mathrm{PV})$, que foi de $\mathrm{R} \$ 15,97$, resulta num diferencial de renda de $\mathrm{R} \$ 19,58$ (dezenove reais cinqüenta e oito centavos) por animal; e como o ganho de peso total por animal no tratamento T3 $(0,76 \% \mathrm{PV})$ foi de $37,33 \mathrm{~kg}$, a um custo de $\mathrm{R} \$ 46,72$, e uma receita de $\mathrm{R} \$ 52,27$, isto representa uma renda líquida de $\mathrm{R} \$$ 5,55 (cinco reais e cinqüenta e cinco centavos), a qual comparada com ao prejuízo do tratamento com T1 $(0 \% \mathrm{PV})$, tem-se então um diferencial de renda de R \$ 21,52 (vinte e um reais cinqüenta e dois centavos) por animal.

Como a regressão linear sugere que quanto maior for o nível de fornecimento de concentrado, melhor será a relação receita:despesa. Entretanto, ao fazer comparações entre os resultados financeiros obtidos em cada tratamento, pode-se afirmar que o melhor resultado econômico foi o do tratamento T3 que forneceu $0,76 \%$ do PV em concentrado.

TABELA 6 - Valores médios da receita (R\$/animal), despesa (R \$/animal) e relação receita:despesa dos tratamentos ao final do período experimental.

\begin{tabular}{lccc}
\hline Tratamentos & $\begin{array}{c}\text { Receita } \\
(\mathbf{R} \$ / \text { animal) }\end{array}$ & $\begin{array}{c}\text { Despesa } \\
(\mathbf{R} \$ / \text { animal })\end{array}$ & $\begin{array}{c}\text { Receita: } \\
\text { Despesa }\end{array}$ \\
\hline $0,00 \%$ PV & 2,33 & 18,30 & 0,13 \\
$0,37 \%$ PV & 21,23 & 33,56 & 0,63 \\
$0,76 \%$ PV & 52,27 & 46,72 & 1,12 \\
$0,87 \%$ PV & 54,37 & 50,76 & 1,07 \\
\hline
\end{tabular}




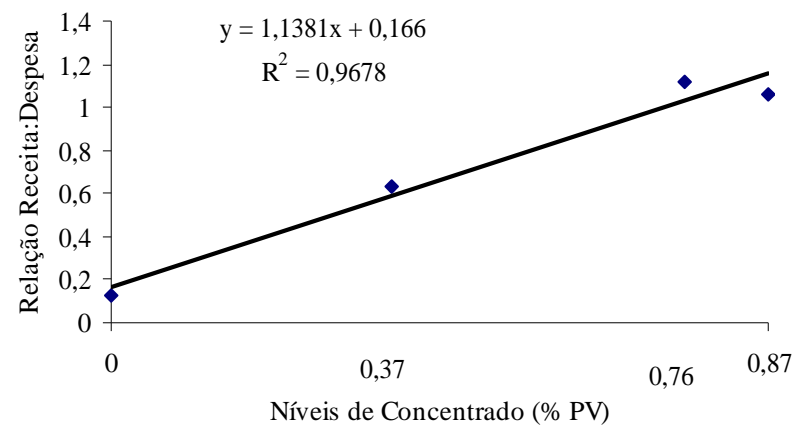

FIGURA 2 - Efeito dos níveis de concentrado na relação Receita: Despesa.

\section{CONCLUSÃO}

O desempenho de novilhos mestiços nelore suplementados em pastagem no período seco do ano, é técnica e economicamente viável, com melhor desempenho econômico quando se forneceu $0,76 \%$ do peso vivo em concentrado e melhor desempenho biológico quando se forneceu $0,87 \%$ do peso vivo em concentrado, portanto, obteve-se o melhor desempenho bioeconômico quando se forneceu $0,76 \%$ do peso vivo em concentrado.

\section{REFERÊNCIAS BIBLIOGRÁFICAS}

ALMEIDA, A. J. de; AZEVEDO, C. Semiconfinamento. São Paulo: Globo, 1999. 184 p.

ANUALPEC. Anuário estatístico da pecuária de corte. São Paulo: FNP Consultoria \& Comércio, 2001. 250 p.

BOIN, C.; TEDESCHI, L. O. Sistemas intensivos de produção de carne bovina: II. crescimento e acabamento In: SIMPÓSIO SOBRE PECUÁRIA DE CORTE, 4., 1997, Piracicaba. Anais... Piracicaba: FEALQ, 1997. p. 205-228.

BOMFIM, M. A. D. Níveis de concentrado na terminação de novilhos Holandês $x$ Zebu suplementados a pasto na estação seca. 2000. 62 p. Dissertação (Mestrado em zootecnia) - Universidade Federal de Lavras, Lavras, 2000.

CASTRO NETO, P.; SEDIYMA, G. C.; VILELA, E. A. Probabilidade de ocorrência de períodos secos em Lavras, Minas Gerais. Ciência e prática, Lavras, v. 4, n. 1, p. 46-55, 1980.
EUCLIDES, V. P. B.; MACEDO, M. C. M.; OLIVEIRA, M. P. Produção de bovinos em pastagens de Brachiaria spp. consorciadas com Calopogonium mucunoides nos cerrados. Revista Brasileira de Zootecnia, Viçosa, v. 27, n. 2, p. 238-245, 1998.

FERREIRA, D. N. Análises estatísticas por meio do Sisvar para Windows versão 4.0. In: REUNIÃO ANUAL DA REGIÃO BRASILEIRA DA SOCIEDADE INTERNACIONAL DE BIOMETRIA, 2000, São Carlos. Anais... São Carlos: UFSCar, 2000. p. 225-258.

GUIDONI, A. L. Alternativas para comparar tratamentos envolvendo o desempenho nutricional animal. 1994. $105 \mathrm{f}$. Tese (Doutorado em Estatística e Experimentação Agronômica) - Escola Superior de Agricultura de Luiz de Queiroz, Piracicaba, 1994.

KÖEPPEN, W. Climatologia. Buenos Aires: Panamericana, 1948. $478 \mathrm{p}$.

PAULINO, M. F. Misturas múltiplas na nutrição de bovinos de corte a pasto. In: SIMPÓSIO GOIANO SOBRE PRODUÇÃO DE BOVINOS DE CORTE, 1999, Goiânia. Anais... Goiânia: UFG, 1999. p. 95-104.

OMETTO, J. C. Bioclimatologia vegetal. São Paulo: Ceres, $1981.440 \mathrm{p}$.

NATIONAL RESEARCH COUNCIL. Nutrients requirements of beff cattle. 7. ed. rev. Washington, 1996. $241 \mathrm{p}$.

SILVA, D. J. Análise de alimentos: métodos químicos e biológicos. Viçosa: UFV, 1998. 165 p.

SOEST, P. J. van; ROBERTSON, J. B.; LEWIS, B. A. Methods for dietary fiber, neutral detergent fiber, and nonstartch polysaccharides in: animal nutrition. Journal of Animal Science, Champaign, v. 74, n. 10, p. 3583-3597, Oct. 1991.

VILELA, E. S.; RAMALHO, M. A. P. Análise das temperaturas e precipitações pluviométricas de Lavras, Minas Gerais. Ciência e Prática, Lavras, v. 3, n. 1, p. 71-79, 1979.

WAGNER, D. G.; GILL, D.; LUSBY, K. Feeding cattle on grass. 1995. Disponível em? $<$ http:/ansi.okstate.edud 'EXTEN/BEEF/FS3011.PDPF>. Acesso em: 7 jan. 2002. 